\title{
Application of 3D Printing Technology in Machining and Manufacturing
}

\author{
Guo Lin \\ School of Mechanical Engineering, Liaoning Equipment Manufacturing Vocational and Technical \\ College, Shenyang, Liaoning, China
}

\begin{abstract}
$3 D$ printing technology is a rapid prototyping technology, which has been gradually applied in the mechanical automation manufacturing industry. 3D printing technology of mechanical parts is an important development direction of advanced mechanical automation manufacturing technology. At present, the research and development of SLM is a new hotspot in the field of rapid prototyping at home and abroad. The application of this technology can not only reduce the processing time, avoid the resource consumption caused by repeated adjustment of parameters, and make the mechanical manufacturing more accurate, more economical and more efficient. Based on this, this paper focuses on the application of $3 D$ printing technology in mechanical manufacturing automation, and takes SLM as the research object, expounds the basic composition and forming principle of SLM. Based on the comparison of SLM technology at home and abroad, the future development direction of SLM technology is analyzed. At the same time, this paper designs SLM equipment with high efficiency, high cost performance, large range and traditional machining methods. The experimental results show that the $3 D$ printing technology of metal parts is an important development direction of advanced mechanical automation manufacturing technology. The development direction of metal $3 D$ printing in the future is to develop a portable and intelligent $3 d s l m$ device with high efficiency, high cost performance, high processing capacity and combination with traditional machining methods.
\end{abstract}

Keywords: $3 D$ printing, rapid prototyping, mechanical parts, SLM.

\section{Introduction}

In modern society, a variety of modern science and technology in the rapid development, at the same time promote the rapid development of related machinery manufacturing industry, now mainly to 3D printing technology as the theme, combined with other optical and electrical knowledge, and constantly meet the diversified needs of large enterprises, but also as far as possible to reduce costs, and constantly improve its operating efficiency [1-2].

As an important link in the development of modern science and technology, the rapid development of 3D printing technology promotes the outbreak of modern scientific and technological revolution, and it is also an important driving force to promote the continuous development and expansion of large enterprises. It is mainly based on modern 3D printing technology to continuously improve the level of mechanical manufacturing and processing. With the rapid development of polymer materials and science and technology, relevant enterprises and government departments constantly adjust the relevant resources, and finally realize the optimal allocation of resources, which brings breakthrough progress to modern industrial technology [3]. At present, China's 3D printing technology is constantly developing and improving, which provides a huge development opportunity for the current machinery manufacturing industry [4-6]. At the same time, the current 3D printing technology in the development process also has some problems and deficiencies, its development is not very perfect, some places can not meet the requirements of businesses, these problems and deficiencies may affect the upgrading and development of the machinery industry to a certain extent [7].

In recent years, with the joint efforts of many experts and scholars, 3D printing technology has developed rapidly, and gradually overcome its own shortcomings. Therefore, China's machinery manufacturing automation industry

ISSN: 0010-8189 
has great development advantages. It can be seen from the following aspects [8]: (1) in today's context of relatively mature science and technology, the technology develops rapidly, is easy to promote, and can promote the rapid development of production; (2) the development of the technology promotes the development of today's society; (3) science and technology uses new technology as the media to promote the change of production relations.

Intelligent development is the mainstream development direction of machinery manufacturing industry. With the application of 3D printing technology, mechanical manufacturing is gradually transforming from traditional manufacturing mode to intelligent direction. For example, in the mechanical manufacturing production, the analysis software is applied to optimize and process the materials and design, and the modeling tools are applied to build the 3D model on the computer platform, and then the optimized model data is transmitted to the 3D printer to realize 3D printing and produce high-quality mechanical products. In this process, the optimization and adjustment of the model can be carried out from time to time, and designers can make multiple choices and adjustments according to the actual situation, making the production of products more selective. At the same time, in the whole process, the intelligent technology can be effectively applied to quickly locate and solve the problems, so as to make the mechanical manufacturing more efficient, more economical and more intelligent, and promote the mechanical manufacturing industry to gradually move towards the direction of intelligent development.

\section{Features and implementation of 3D printing technology}

In recent years, China's mechanical manufacturing automation technology is gradually developing towards intelligent and diversified direction. With the rapid development of modern intelligent technology, 3D printing technology has also obtained a huge development opportunity, in the rapid development. At the beginning, 3D printing technology was mainly developed at the end of the 20th century. In today's society, it has been growing and loved by all walks of life, and more and more excellent 3D works have been produced.

In the process of using 3D printing technology, we can use computer software program as the carrier, use the most advanced science and technology and polymer materials, select the appropriate printing equipment, and use network computer software to transfer data to obtain complete 3D printing information. As a classical mechanical technology, 3D printing technology strictly inherits the essence of traditional manufacturing process, and further develops innovatively, so as to develop 3D printing works that meet people's needs.

3D printing technology is usually applied together with computer technology. After the application, the product parameters can be adjusted according to the personalized needs, and the products with specific structure can be customized according to the needs, so that the customization advantages of 3D printing technology are gradually highlighted, and its development direction is also to be customized. At the same time, with the support of network platform, 3D printing technology can produce rapidly and print with high quality, which provides a broader development space for mechanical manufacturing and automation.

Compared with the traditional mechanical manufacturing, 3D printing technology has certain advantages, mainly reflected in the ecological and energy saving. The application of 3D printing technology can realize the digital manufacturing of products, the environmental protection characteristics of products are more prominent, and the maximum application of raw materials can be realized, so as to reduce the cost and loss, so that $3 \mathrm{D}$ printing technology can get rid of the limitation of material loss and develop in the direction of economy and environmental protection. The ecological characteristics of 3D printing technology will lead the mechanical manufacturing and automation to the ecological direction.

Printing drive control is an important part of 3D printing technology, which can improve the quality of products to a certain extent. In order to better promote the development of this technology, we must clearly understand the internal structure and operation process of printing.

As shown in Figure 1, the frame is made of aluminum profile, and three torchers which can move in a straight line in the vertical direction are installed on the frame column, and the stepping motor drives the pulley to move up and down ${ }^{2-3}$. The slider is connected to the printer nozzle through the fixed length inclined rod, and the three small

ISSN: 0010-8189 
torchers cooperate to move the movement of the trochlear into the horizontal plane of the nozzle. The printer nozzle is the key part of the 3D printing system. It is mainly composed of three parts: the guide part, the heating part and the heat dissipation part. The feeding part is composed of core inserted Teflon (PTFE) pipe and nozzle, the nozzle aperture size directly affects the thickness of silk.

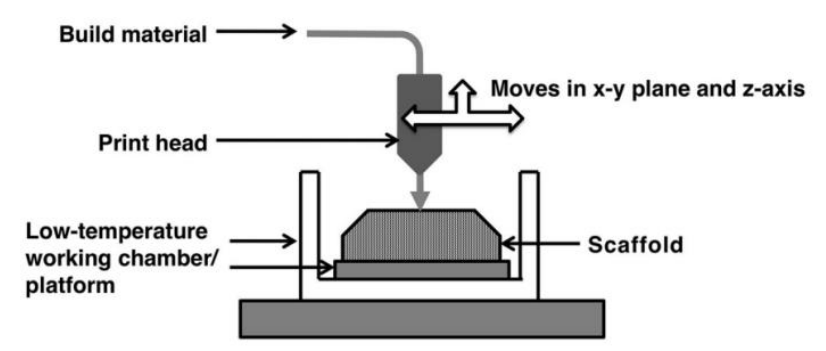

Fig 1: Scheme of $3 D$ printer

We have a structural design for the mechanical structure. Pre-tightening force of each bolt [9-10]:

$$
F_{0}>\frac{K_{s} F_{\Sigma}}{f i z}
$$

The pre-tightening force of each bolt:

$$
F_{0} \geq \frac{K_{s} T}{f \sum_{i=1}^{n} r_{i}}
$$

The position of the 3D printer's nozzle in space is obtained by the distance of the three torchers to coordinate the moving distance. Loose bolt connection:

$$
\sigma=\frac{F}{\pi d_{1}^{2} / 4} \leq[\sigma]
$$

In a triangle composed of three dimensions, which are projected in a horizontal and vertical direction, the length of the inclined rod is unchanged. Tight bolt connection:

$$
\sigma=\frac{1.3 F_{0}}{\pi d_{1}^{2} / 4} \leq[\sigma]
$$

Suppose the nozzle moves only on the $\mathrm{X}$ axis and does not move on the $\mathrm{Y}$ axis and the $\mathrm{Z}$ axis. Axial static load:

$$
\sigma=\frac{1.3 F_{2}}{\pi d_{1}^{2} / 4} \leq[\sigma]
$$

Axial dynamic load:

$$
\sigma_{a}=\frac{C_{b}}{C_{b}+C_{m}} \cdot \frac{2 F}{\pi d_{1}^{2}} \leq\left[\sigma_{p}\right]
$$

Shear strength conditions of bolts:

ISSN: 0010-8189 


$$
\tau=\frac{F}{\pi d_{0}^{2} / 4} \leq[\sigma]
$$

Extrusion strength of bolt and wall:

$$
\sigma_{p}=\frac{F}{d_{0} L_{\min }} \leq\left[\sigma_{p}\right]
$$

In the 3D printer, the length of the diagonal bar is one of the main factors that affect the printing quality. If the actual length of the horizontal projection of the inclined rod is equal to the theoretical length, the actual length of the inclined rod is larger than the theoretical length, and the equation trajectory becomes a larger circle in the graph as shown in Figure 2.

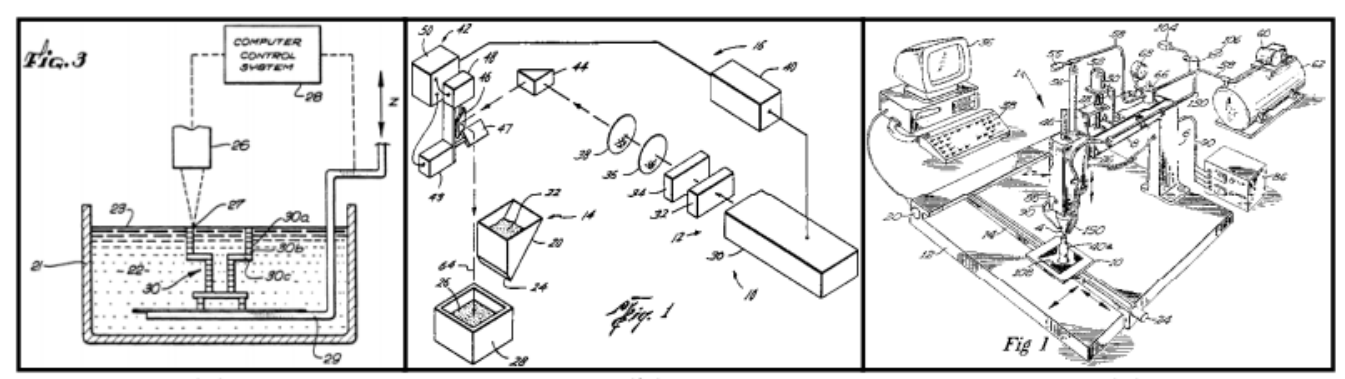

(a)

(b)

(c)

Fig 2: 3D printing related patent diagram

As we can see in Figure.3, the error of oblique rod length can be corrected by comparing the measured length and design size of printing models, such as printing regular cuboid model, measuring its length and calculating printing error, correcting the length of diagonal bar according to the error value.

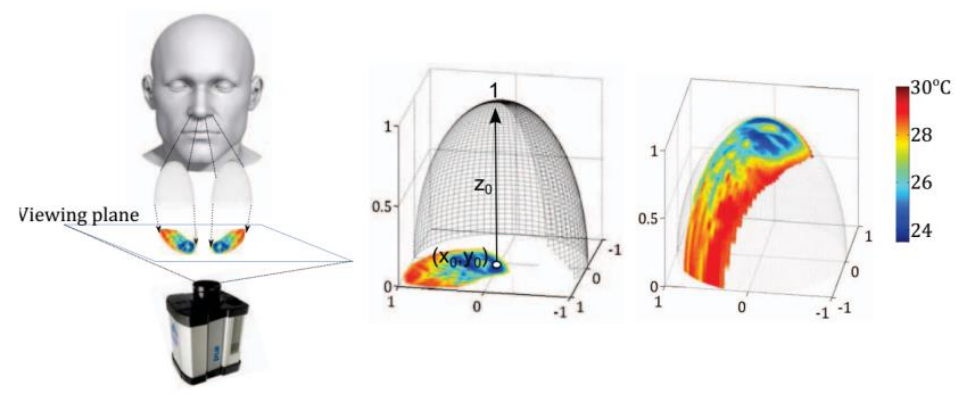

Fig 3: Experimental layout

Horizontal deviation of the sprinklers to $\mathrm{Z}$ direction, resulting in a concave deviation bowl to print a plane. On the other hand, if the deviation of the movement is small, a convex deviation is produced to make the print plane into the dome as shown in Figure 4.

ISSN: 0010-8189 


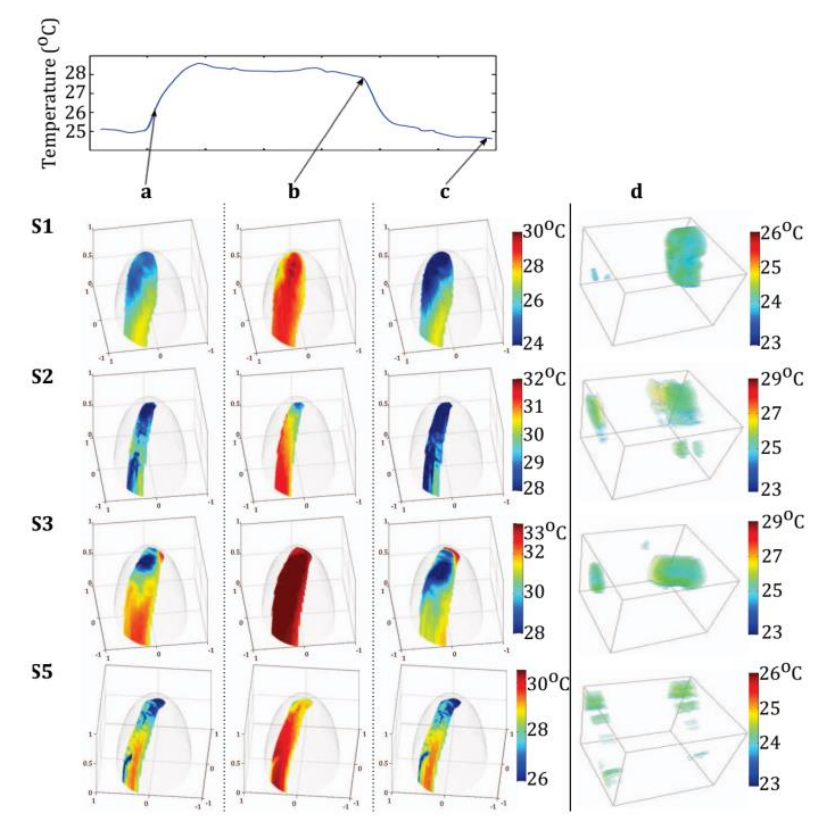

Fig 4: Normal breathing cycles on the left nostrils of four subjects

\section{Basic composition and implementation of SLM}

3D printing technology of metal parts is an important development direction of advanced mechanical automation manufacturing technology. Metal 3D printing technology is divided into three categories:

(1) A laser is used to irradiate the pre spread metal powder. It includes selective laser melting (SLM) and direct metal laser sintering (DMLS). Among them, the laser selective melting technology is relatively mature at present, which can be directly processed for almost any shape parts, and the density can reach nearly $100 \%$. (2) A laser is used to irradiate the powder stream delivered by the nozzle, and the laser works simultaneously with the delivered powder (lens). This method is widely used in China. (3) Electron beam is used to melt pre spread metal powder (EBM). This method is similar to the first principle except that the heat source used is different.

3D Model of ceramic ring is shown in Figure 5. SLM is generally composed of mechanical unit, control unit, light path unit, protective gas seal unit and process software:

(1) The optical path unit mainly includes reflector, fiber laser, focusing lens, scanning galvanometer and beam expander. Laser is the core part of SLM equipment. In recent years, almost all SLM equipment use fiber laser. Fiber laser has the advantages of high conversion efficiency, reliable performance, long life, and beam mode close to the fundamental mode. Because of the good beam quality of fiber laser, fiber laser has obvious advantages in laser selective melting rapid prototyping of precision metal parts. The scanning galvanometer is driven by a motor and controlled by a computer, so that the laser spot can be accurately located at any position on the machining surface.

(2) The mechanical unit mainly includes molding red, molding chamber sealing equipment, powder red, powder spreading device, etc.

(3) The control system is composed of a computer and several control cards. The laser beam scanning control is that the computer sends a control signal to the scanning galvanometer through the control card to control the movement of the $\mathrm{X} / \mathrm{y}$ scanning mirror to realize laser scanning. The equipment control system mainly includes the following functions:

1) System initialization, state information processing, fault diagnosis and human-computer interaction function;

ISSN: 0010-8189 
2) The motor system is controlled in various ways, and the motion control of forming piston, powder feeding piston and powder roller is provided;

3) To control the scanning galvanometer, set the moving speed and scanning delay of the scanning galvanometer;

4) Setting various parameters of automatic forming equipment, such as adjusting laser power, forming red, powder spreading cylinder rising and falling parameters, etc.

5) Coordinated control of five motors, and then control the parts of the molding process. According to the needs of SLM process, there are three kinds of professional software involved: slicing software, scanning path generation software and equipment control software.
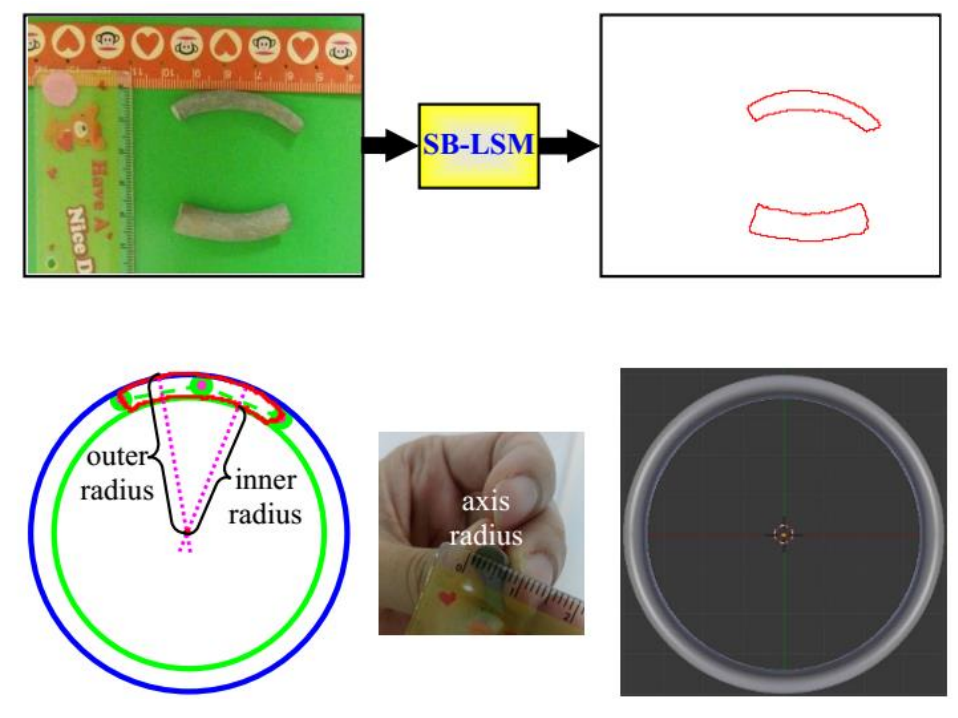

Fig 5: 3D Model of ceramic ring

\section{Conclusion}

With the development of machinery manufacturing industry and automation, the social productivity has been greatly improved, and the traditional machinery manufacturing mode has been unable to adapt to the society. We must introduce innovative mechanism in the design and realize the change of concept, so as to guide the machinery manufacturing industry to develop in the direction of environmental protection, energy saving, high efficiency, intelligence and sustainability. In the development of mechanical manufacturing and automation, the production of mechanical parts requires enterprises to take quality as the goal and quantity as the breakthrough. At this time, the enterprise must innovate in the design concept, and use intelligent production technology to guarantee the quality and quantity. The application of 3D printing technology can make use of multi-dimensional concept, intelligent concept, environmental protection concept and scientific development concept in product design to provide spiritual innovation planning for products, effectively solve the problems in production, and achieve the goal of saving energy, reducing consumption and improving economic benefits. By optimizing the process, the cost of 3D printing is effectively controlled, the production process is easy to operate, and the product value is improved. Guiding the mechanical manufacturing and automation to continuously optimize the process, innovate the design concept, and realize the sustainable intelligent development.

\section{References}

[1] Xiao Li, Bai Xiao, Li Qinglian. Comparative Study on Atomization Characteristics of 3d Printing and Machining Nozzles. Rocket Propulsion, 2020, V.46; No.233 (01): 38-46

[2] Liu Meng. Research on the Application of 3d Printing Slm Technology in Mechanical Automatic Processing. Digital Technology and Application, 2015 (4): 107-107

ISSN: 0010-8189 
[3] Jiang Long, Yao Xiaotong. Analysis of 3d Printing Forming Method and Its Advantages in Machining and Manufacturing. Internal Combustion Engine and Accessories, 2020, No. 323 (23): 118-119

[4] Liang Yi Yong. Discussion on the Application of 3d Printing Technology in Petroleum Machining Parts. Automation Today, 2019, 4: P.204-205

[5] Wei Xinglei. Application of 3d Printing Technology in Machinery Manufacturing. Hebei Agricultural Machinery, 2019, No. 252 (06): 44-44

[6] Chen Ziheng, Li Xinmin, Hui Kunlong. Research on Application of 3d Printing Technology in Difficult to Machine Parts of Petroleum Machinery. Forging Equipment and Manufacturing Technology, 2018, V.53; No.315 (03): 90-92

[7] Feng Xiaohui. Research on Mechanical Automatic Processing and Application Based on 3d Printing Slm Technology. Digital Communication World, 2019, $10: 191-197$

[8] Ma Xiaowei. on the Application of 3d Printing Technology in the Field of Machinery Manufacturing. China's Strategic Emerging Industries (theoretical Edition), 2019, 000 (001): 1-1

[9] Zhang Yue. Research on 3d Printing Technology and Its Application Progress. Journal of Central China Normal University, Natural Science Edition, 2016 (04): 11-13

[10] Wang Zeyuan, Zhou Yufeng, Ji Fangqiu. Finite Element Analysis of One-stage Implant Stability Based on 3d Printing. Software, 2020, 041 (002): 246-251 